\title{
Investigation of Thermal Effects in Coaxial Probe Method and Dielectric Characterization of Glycerol up to $140^{\circ} \mathrm{C}$
}

\author{
Deibnasser C. Campos ${ }^{1}$ (D, José C. A. Santos ${ }^{2}$ (E) Luiz E. P. Borges ${ }^{1}$ (i) \\ Instituto Militar de Engenharia - IME, Praça General Tibúrcio, 80, Urca, 22290-270, Rio de Janeiro - RJ \\ ${ }^{1}$ Seção de Engenharia Química, deibnasser@gmail.com,luiz@ime.eb.br \\ ${ }^{2}$ Seção de Engenharia Elétrica, araujo@ime.eb.br
}

\begin{abstract}
Dielectric properties of a material are of paramount importance in many areas of knowledge. All scientific fields that deal with the interaction of electromagnetic radiation with matter demand this information. Consequently, suitable methods for obtaining accurate dielectric data are required. In the microwave community, the dielectric metrology with the coaxial probe method is well known due to its simplicity and practicality. It is also pointed out as a broadband and wide temperature range technique. Despite this, a detailed study of the implications of thermal effects at calibration with this technique has not been plainly reported in the literature. In the present work, a concise exposition of the problem is made. Using air, short and water as standards, we have analyzed a set of calibration procedures with different thermal conditions and compared them with the isothermal case. With the aid of statistical analysis, we have made recommendations for precise and accurate dielectric measurements at high temperatures. Using glycerol as material under test (MUT) we also demonstrated that measurements at temperatures up to $50^{\circ} \mathrm{C}$ above the calibration temperature can provide reliable data. Thus, measurements of glycerol up to $140^{\circ} \mathrm{C}$ were performed by calibrating the system with water at $90^{\circ} \mathrm{C}$. This important issue was raised due to the lack of standard material data in the literature for temperatures above $100^{\circ} \mathrm{C}$. From the measured data we also have derived the ColeDavidson model parameters of glycerol from 30 to $140^{\circ} \mathrm{C}$.
\end{abstract}

Index Terms - Coaxial Probe, Dielectric Characterization, Glycerol, High Temperature Permittivity Measurements

\section{INTRODUCTION}

The coaxial probe technique became popular in recent years as a versatile permittivity measurement method. It is non-destructive, and so it is widely used in medical fields to obtain dielectric parameters of biological tissues [1]. It is broadband, and operates in a large temperature range [2]. Specially designed probes can perform measurements up to $270^{\circ} \mathrm{C}$ [3] and 180 bar [4].

The method itself is relatively simple. The coaxial probe, which is a truncated transmission line, is placed in contact with the material under test (MUT). From the reference plane (interface between the probe and MUT), the reflection coefficient is measured. Then mathematical models, such as 
capacitive circuit, rational function, variational technique, between others, can be used to compute the material permittivity from the measured reflection coefficient [5], [6].

The most complex part of the method is the calibration procedure, necessary to remove systematic errors from the measurement setup. For a typical reflection coefficient measurement with a network analyzer, a one-port error model is used whose error terms are directivity, source match and reflection tracking. These terms are derived from general equations, which can be solved from three simultaneous equations with three unknowns. Usually three well characterized materials are used to build these equations and provide the correction terms, allowing the user to obtain the permittivity from the corrected reflection coefficient from any material after calibration [7]. In principle, one may use any material as calibration standard, provided its dielectric properties are well known. A common trio is open (air), short (metal) and load (a well characterized material). If the load is liquid, it is also referred to as reference liquid (RL) [8]. Among these three standards, the easier to measures are for open and short. For the open (air), the surface of the probe must be properly cleaned for reliable measurements [9]. In the case of short, a good conductor must be used as standard, and the contact metal surface should be flat and free of rust or scratches.

The most laborious step in the calibration process is for the load, whose material must have several properties, such as availability, high purity, innocuousness, chemical inertness, between others [9]. Many efforts have been devoted to the selection and characterization of liquids that meet these requirements at a wide range of frequencies and temperatures [8], [10]. Currently the only liquid that nearly meets all those requirements is water [9], [11], for which dielectric data are well characterized [12].

Performing measurements at temperatures other than room temperature requires, in principle, calibration of the dielectric measurement system at the same temperature of measurement. When there are differences between calibration standard and MUT temperatures, protrusion or receding of the probe bead can occur [13], changing the probe electrical properties, and therefore the value of the measured reflection coefficient. Reference [14] shows that a difference of -5 to $15^{\circ} \mathrm{C}$ between calibration and measurement temperatures does not cause significant errors in the actual value of the measured permittivity. Concerning the temperature of each standard, [9] reports that you can leave only the reference liquid at the temperature of measurement, with no evidence that it is effective. An assessment carried out by [15] demonstrates that the relative error of measurement increases with the thermal difference between the calibration temperature $\left(\mathrm{T}_{c a l}\right)$ and the measurement temperature $\left(\mathrm{T}_{\text {meas }}\right.$ ), and that it is more significant for $\varepsilon^{\prime \prime}$ than for $\varepsilon^{\prime}$. Obviously, the closer $\mathrm{T}_{\text {cal }}$ is to $\mathrm{T}_{\text {meas }}$, the less the error will be. Furthermore, [15] also states that a detailed description of this method has not yet been reported in the literature. Reference [16] makes an assessment to the accuracy of the method using water and methanol as MUT, also claiming difficulties in finding a detailed description of the method. Until the present date, to the best of our knowledge, the previous statements remain true. 
A more careful and elaborated calibration procedure for thermal measurements then becomes necessary. To achieve this goal, firstly a proper description of the problem is necessary.

The calibration technique in question is the tri-standard calibration: open, short and RL. The calibrated system is used to measure any material (MUT). Let the standard temperatures be $T_{1}, T_{2}$, and $\mathrm{T}_{3}$ (the calibration order of each standard is arbitrary). Also let the MUT temperature be $\mathrm{T}_{\mathrm{MUT}}$. Consider the following thermal combinations:

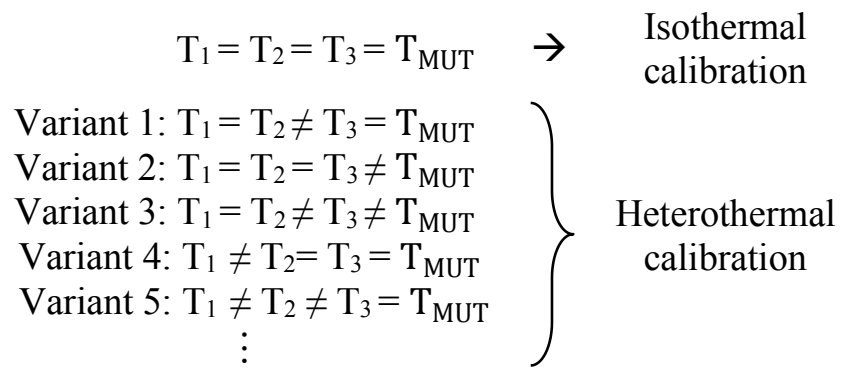

In principle, isothermal calibration should produce the best results, since the thermal expansion of the probe constituent materials would remain constant from calibration to measurement. For this, it is necessary to measure every calibration standard at the measurement temperature, making data acquisition more time consuming and experimentally costly. Thus, variants that may be equivalent to the isothermal condition are desirable from a practical point of view. In this work, heterothermal variant 1, 2 and 3 calibrations are investigated and compared with the isothermal case, since they are faster and require less complex apparatus or equipment, such as temperature controllers, samplers and microclimatic chambers [15]. The remaining heterothermal variants are not suitable from this point of view. In [17], measurements were performed with variant 1, using a thermostatic bath for the RL and another for the MUT to decrease the measurement time. In terms of experimental apparatus, variant 3 is identical to 1 . Therefore, variant 2 is the most attractive, since calibration can be performed with all standards at the same temperature (eventually at ambient or room temperature), and only the MUT should be heated or cooled.

Another open question related to this matter is the need to recalibrate the system after a number of measurements runs. In this respect, a detailed description was not found in the literature, especially regarding to heterothermal measurements. Reference [18] suggests that only one calibration is adequate. However, [9] states that errors in calibration propagate to subsequent measurements, and recommends that the calibration be always verified. This subject becomes non-trivial when dealing with measurements at high temperatures, and even more crucial if the dielectric data of the material under test are poorly known (or nonexistent) in literature. This is the case of reaction mixtures for instance [17], [19].

The present work aims at the investigation of temperature effects on the coaxial probe tri-standard calibration method with the aid of statistical analysis. Section II presents the used materials and methods. Section III presents the measured results and the discussion about them. Section IV presents the final considerations of the work. 


\section{MATERIAL AND METHODS}

Permittivity is a property that quantifies the response of a material in the presence of an electric field. The commonly used symbol to represent permittivity is $\varepsilon$. The latter is an absolute value that has the unit of $\mathrm{F} / \mathrm{m}$ (Faraday per meter). When related to vacuum permittivity $\left(\varepsilon_{0}\right)$, we have the relative permittivity, $\varepsilon_{r}=\varepsilon / \varepsilon_{0}$, also called the dielectric constant of the material. For harmonic fields, the relative permittivity has a complex form $\left(\varepsilon_{r}^{*}\right)$ and consequently has real $\left(\varepsilon_{r}^{\prime}\right)$ and imaginary $\left(\varepsilon_{r}^{\prime \prime}\right)$ parts, so that $\varepsilon_{r}^{*}=\varepsilon_{r}^{\prime}-j \varepsilon_{r}^{\prime \prime}$. The imaginary part accounts for the dissipation phenomenon in the material. For the sake of textual conciseness, the real and imaginary parts of complex relative permittivity will be written as $\varepsilon^{\prime}$ and $\varepsilon^{\prime \prime}$, respectively, so that in our notation we will use: $\varepsilon=\varepsilon^{\prime}-j \varepsilon^{\prime \prime}$.

The dielectric measurements were performed with a network analyzer Agilent E5070B ENA $300 \mathrm{KHz}-3 \mathrm{GHz}$, an Agilent 85070E calibration kit, and the software 85070 Version E1.00, also from Agilent Technologies (currently Keysight). In our experiments, distilled water was used as RL (because it already has its permittivity values in several temperatures embedded in the software) and glycerol P.A (99.5\%) as MUT. We used 281 frequency points from 0.2 to $3 \mathrm{GHz}$. Fig. 1 shows the measurement setup.

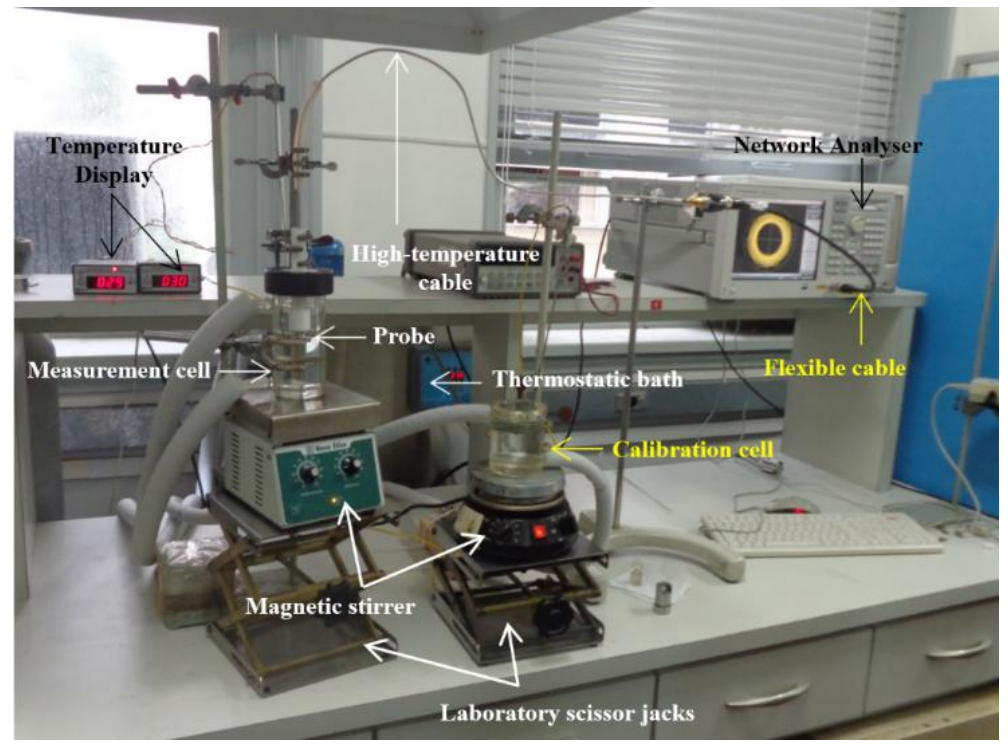

Fig. 1. Dielectric properties measurement system.

The statistical test to determine the equivalence between the heterothermal variants was the Student's $t$-test [20]. For it to be used properly, a prior $F$-test was used to determine whether the data have the same variances [21]. For the aforementioned tests to be applied, the hypothesis of normality of data was investigated. The Ryan-Joiner normality test [21] was applied to evaluate this condition.

The proportion between the number of calibrations and the number of measurements (defined as PCalMeas in this work) is also investigated. Thus PCalMeas equal to $1: n$ means that the system was calibrated once and $n$ measurements were made using that calibration. PCalMeas equal to 1:1 means that just one measurement was made after calibration. The $F$-test was used in this analysis.

All statistical tests used the confidence level of $95 \%(0.95$, or significance level $\alpha=0.05)$. The 
critical values $\left(\mathrm{C}_{\mathrm{V}}\right)$ were obtained from [22]. The $Q$-test [20] was used to deal with outliers.

Measurements were made with the thermal conditions of Table I, which are labeled with the notation of previous section for the thermal combinations. We have used the built-in standard values of the software Agilent 85070 to set the permittivity values of water at different temperatures for calibration.

\begin{tabular}{|c|c|c|c|c|}
\hline & \multicolumn{3}{|c|}{$\begin{array}{c}\text { Calibration Standards } \\
\text { (air, short, and water, in this order) }\end{array}$} & \multirow{2}{*}{$\begin{array}{c}\begin{array}{c}\text { MUT } \\
\text { (glycerol) }\end{array} \\
\mathrm{T}_{\text {MUT }}\end{array}$} \\
\hline & $\mathrm{T}_{1}$ & $\mathrm{~T}_{2}$ & $\mathrm{~T}_{3}$ & \\
\hline Isothermal & $30 ; 90$ & $30 ; 90$ & $30 ; 90$ & $30 ; 90$ \\
\hline Variant 1A & room & room & 50 & 50 \\
\hline Variant 1B & room & room & $30 ; 90$ & $30 ; 90$ \\
\hline Variant $1 \mathrm{C}$ & room & room & $30 ; 60$ & $30 ; 60$ \\
\hline Variant 1D & room & room & $30-90$ & $30-90$ \\
\hline Variant 2 & 20 & 20 & 20 & $30-70$ \\
\hline Variant 3 & room & room & 90 & $100-140$ \\
\hline
\end{tabular}

Note: Room temperature was $\sim 22^{\circ} \mathrm{C} \pm 2{ }^{\circ} \mathrm{C}$.

Twenty-five replicates using heterothermal variant 1A calibration and PCalMeas equal to 1:1 were used in section III-A for the Ryan-Joiner normality test. In section III-B, with variant $1 \mathrm{C}$, a comparison between PCalMeas 1: $n$ and PCalMeas 1:1 was performed. The $t$-test was performed to compare the isothermal and heterothermal variant $1 \mathrm{~B}$ calibrations in section III-C, assuming that the data follow a normal distribution. In section III-D, the data from variant $1 \mathrm{D}$ from 30 to $70^{\circ} \mathrm{C}$ were compared to those of variant 2. Finally, considering the discussion in section III-E, the measured data from variant $1 \mathrm{D}$ and variant 3 were used to derive the Cole-Davidson parameters for glycerol from 30 to $140^{\circ} \mathrm{C}$.

\section{RESULTS AND DISCUSSION}

\section{A. Ryan-Joiner Normality Test}

Using variant $1 \mathrm{~A}$ from Table I, twenty-five replicates were taken to build the probability plots of $\varepsilon^{\prime}$ and $\varepsilon^{\prime \prime}$. Fig. 2 illustrates these data and the linearization performed at $1 \mathrm{GHz}$. Graphics for other frequencies showed similar behavior and were omitted from the text. The linear fit provided the values for the correlation coefficients of Table II for a selected set of frequencies. 

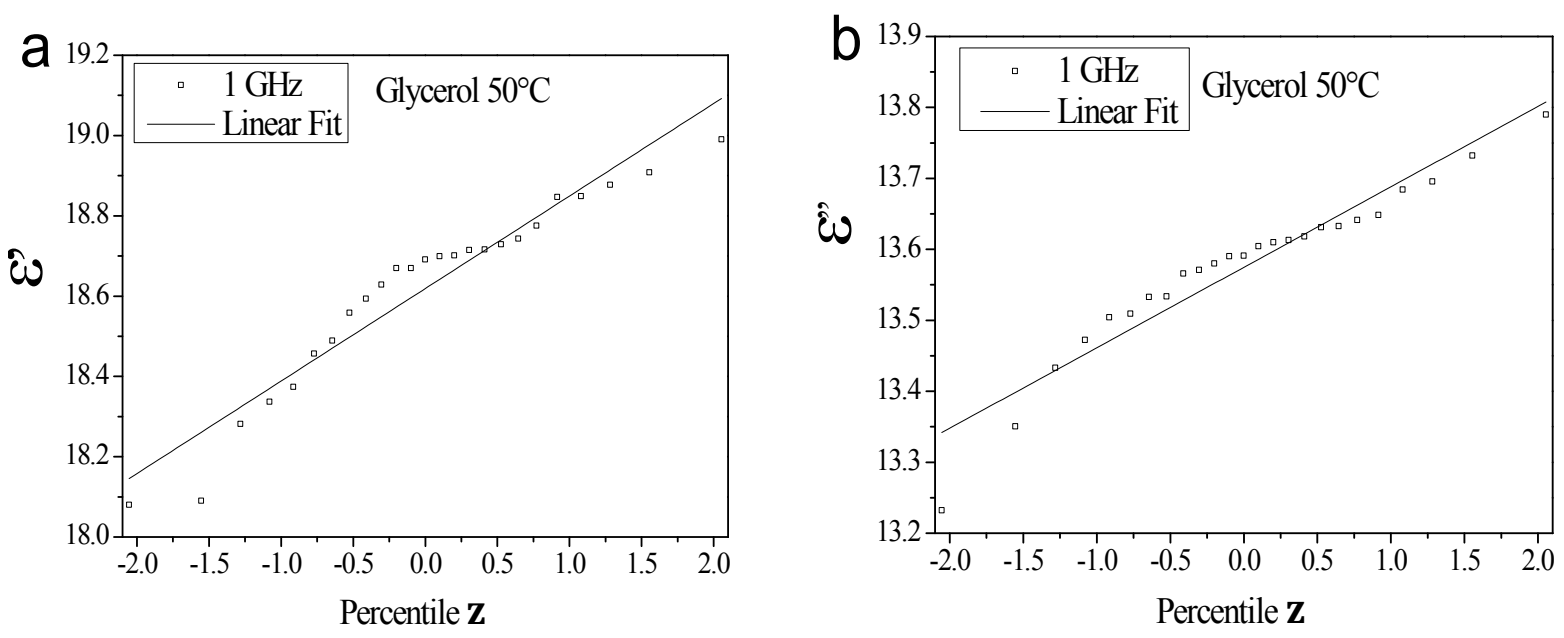

Fig. 2. Probability plot of glycerol showing (a) real and (b) imaginary parts of $\varepsilon$.

TABLE II. CORRELATION COEFFICIENT AS A FUNCTION OF FREQUENCY

\begin{tabular}{ccc}
\hline \multirow{2}{*}{ Frequency $(\mathrm{GHz})$} & \multicolumn{2}{c}{ Correlation coefficient $(r)$} \\
\cline { 2 - 3 }$\varepsilon^{\prime}$ & $\varepsilon^{\prime \prime}$ \\
\hline 0.2 & 0.9911 & 0.9441 \\
0.3 & 0.9680 & 0.9587 \\
0.4 & 0.9454 & 0.9714 \\
0.5 & 0.9457 & 0.9789 \\
0.6 & 0.9455 & 0.9840 \\
0.7 & 0.9447 & 0.9940 \\
0.8 & 0.9635 & 0.9754 \\
0.9 & 0.9732 & 0.9659 \\
1.0 & 0.9638 & 0.9610 \\
2.0 & 0.9909 & 0.9468 \\
3.0 & 0.9862 & 0.9553 \\
\hline $\mathrm{N}=25 ; \alpha=0.05 ; \mathrm{C}_{\mathrm{V}}=0.9582$
\end{tabular}

Comparing the calculated values with the critical Ryan- Joiner value $\left(\mathrm{C}_{V}=0.9582\right)$, it can be concluded that most of the frequency points follow a normal distribution. Some points (4 points for $\varepsilon^{\prime}$ and 3 points for $\left.\varepsilon^{\prime \prime}\right)$, however, are below the critical value.

From the statistical theory, the hypothesis that the data follow a normal distribution should be discarded. However, a more detailed examination shows that the most dissonant point $(0.9441$ in 0.2 $\mathrm{GHz}$ for $\left.\varepsilon^{\prime \prime}\right)$ deviates less than $1.5 \%$ from the critical value. Since all normality deviations are smaller than that value, the hypothesis of normality is still applicable. So, it will be assumed that the data follow a normal distribution.

The test was performed only at the temperature of $50^{\circ} \mathrm{C}$. However, based on the dielectric behavior of glycerol, which has essentially the same temperature and frequency dependence (Debye behavior), it can be assumed that the data are also normal at other temperatures.

\section{B. Comparison Between the Results for PCalMeas 1:n and PCalMeas 1:1}

As stated before, PCalMeas is defined in this work as the proportion between the number of calibrations and the number of measurements for a given measurement scheme. The one-sided $F$-test 
was used to compare the precision for different values of PCalMeas using the variant $1 \mathrm{C}$ condition from Table I. The test result of Fig. 3 shows that for 30 and $60^{\circ} \mathrm{C}$ the variance of PCalMeas 1:1 is always greater than the PCalMeas 1:n. In the figure, $F_{\text {calculated }}$, defined as the ratio between the variance of PCalMeas 1:1 to the variance of PCalMeas 1:n, is always above $F_{\text {critical }}$ (the critical value of the $F$ - test). This indicates that more precise results are obtained when various measurements are performed with the same calibration rather than when one calibration is used for each measurement. These results are in tune with the observed in [9], that although making PCalMeas 1:1 is a more prudent approach, particularly for MUTs with unknown dielectric values, it is less precise.

It is worth mentioning that despite PCalMeas $1: n$ is more precise than PCalMeas $1: 1$, this does not mean that it is more accurate. This is illustrated in Fig. 4, where we present the measurement results of 4 independent calibrations. One of them shows a trend to a second relaxation (refer to the inset of Fig. 4b), which simply is non-existent (which can be checked in Fig. 10), and this behavior is present in all replicates related to this calibration. The obvious and clear conclusion about it is that if the calibration is wrong all subsequent measurements will be as well.
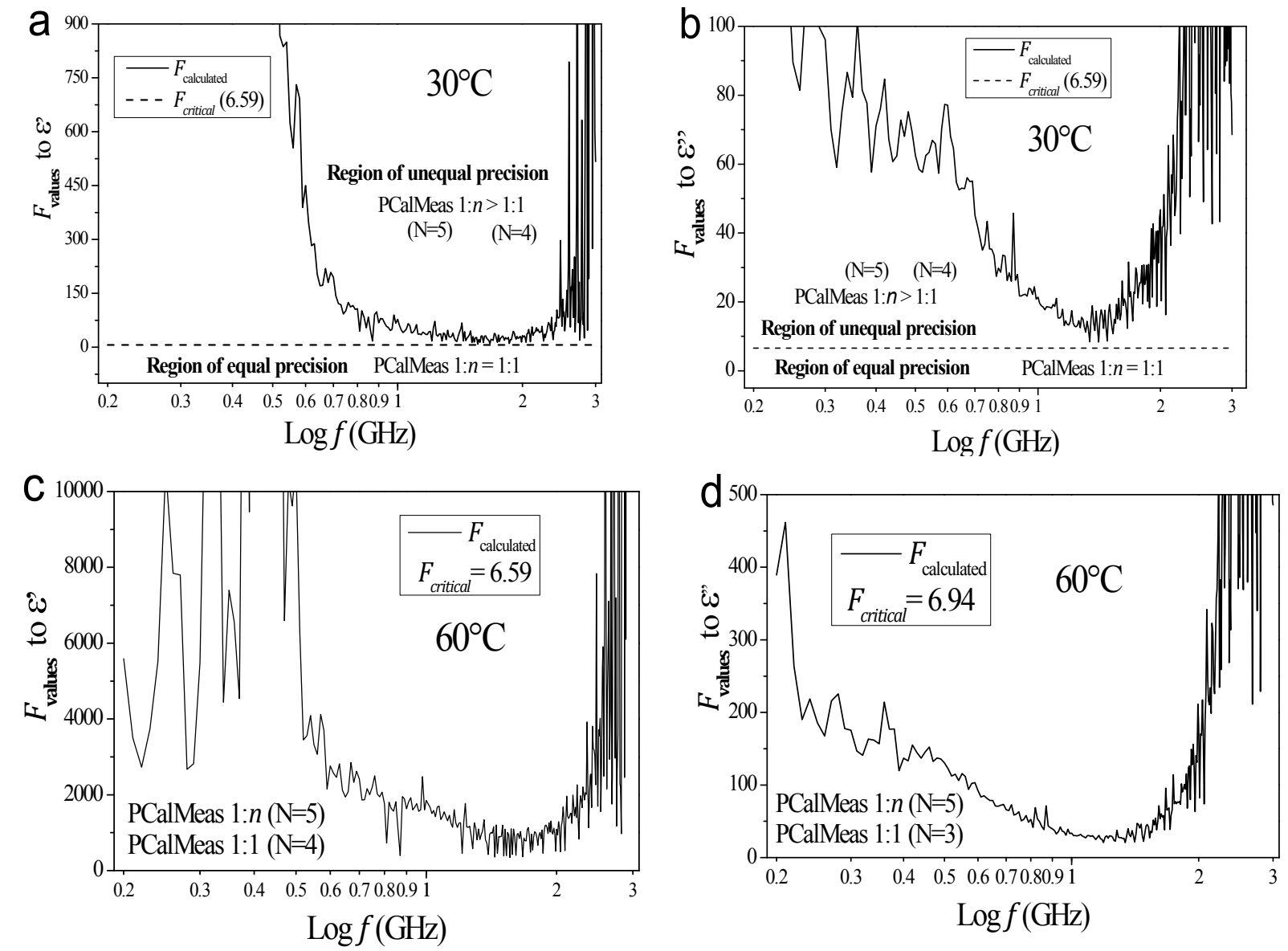

Fig. 3. Comparison between variances of PCalMeas, showing the results of (a) $\varepsilon^{\prime}$ and (b) $\varepsilon^{\prime \prime}$ at $30^{\circ} \mathrm{C}$, and (c) $\varepsilon^{\prime}$ and (d) $\varepsilon^{\prime \prime}$ at $60^{\circ} \mathrm{C} . \mathrm{N}$ is the number of replicates. 

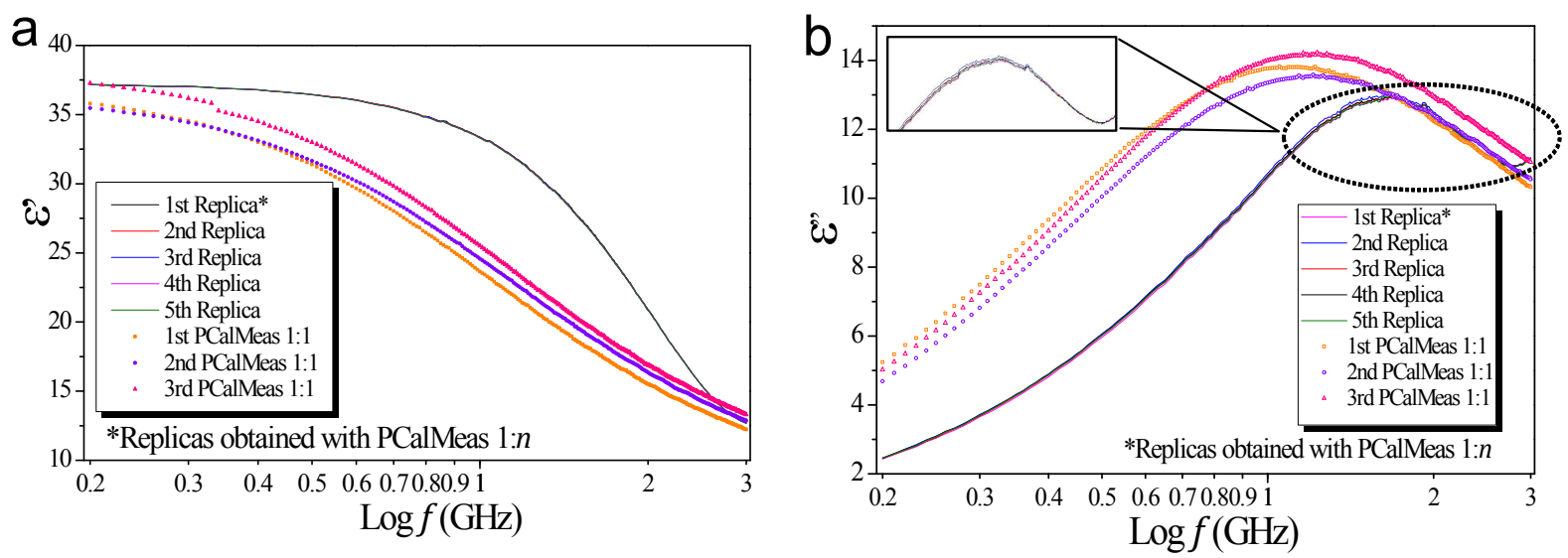

Fig. 4. Comparison between replicas obtained with PCalMeas 1:n and 1:1 of Glycerol at $60^{\circ} \mathrm{C}$.

Regarding runtime, PCalMeas 1:1 is much more sluggish than PCalMeas $1: n$, so it would be interesting to keep the latter scheme. One way to overcome this impasse is to check the accuracy of PCalMeas $1: n$ the first time any measurement is made. For example, just after calibration, the metrologist could measure a reference (or verification) material and compare its results with data either from literature or from previous measurements. In this process it is mandatory the use a material different from the calibration standards. Checking should also be done from time to time at any indication of deviation from the expected behavior of the measured data. To generate verification data, particularly for temperatures above $100^{\circ} \mathrm{C}$, measurements should be done on replicas and their data submitted to the $Q$-test to improve the quality of the results.

\section{Comparison Between Isothermal and Heterothermal Variant 1 Calibrations}

At the isothermal procedure, the standards and MUT are measured at the same temperature. For the heterothermal variant 1 calibration, the first two standards (air and short) are measured at room temperature $\left(\sim 22^{\circ} \mathrm{C}\right)$ and only the reference liquid $\mathrm{RL}$ is set at $\mathrm{T}_{\text {meas }}$. We have used the data from the isothermal and variant 1B calibrations of Table I to compare both procedures (with PCalMeas 1:1). The $F$-test was applied to determine the equality of variance between these calibrations. The twosided $F$-test was performed at 30 and $90^{\circ} \mathrm{C}$, and the results are shown in Fig. 5 (the number of replicas $N$ for each case is stated in the figure).

As can be noticed, almost all spectral points are in the acceptance region, so it can be concluded that both variances (according to precision) are equivalent. 

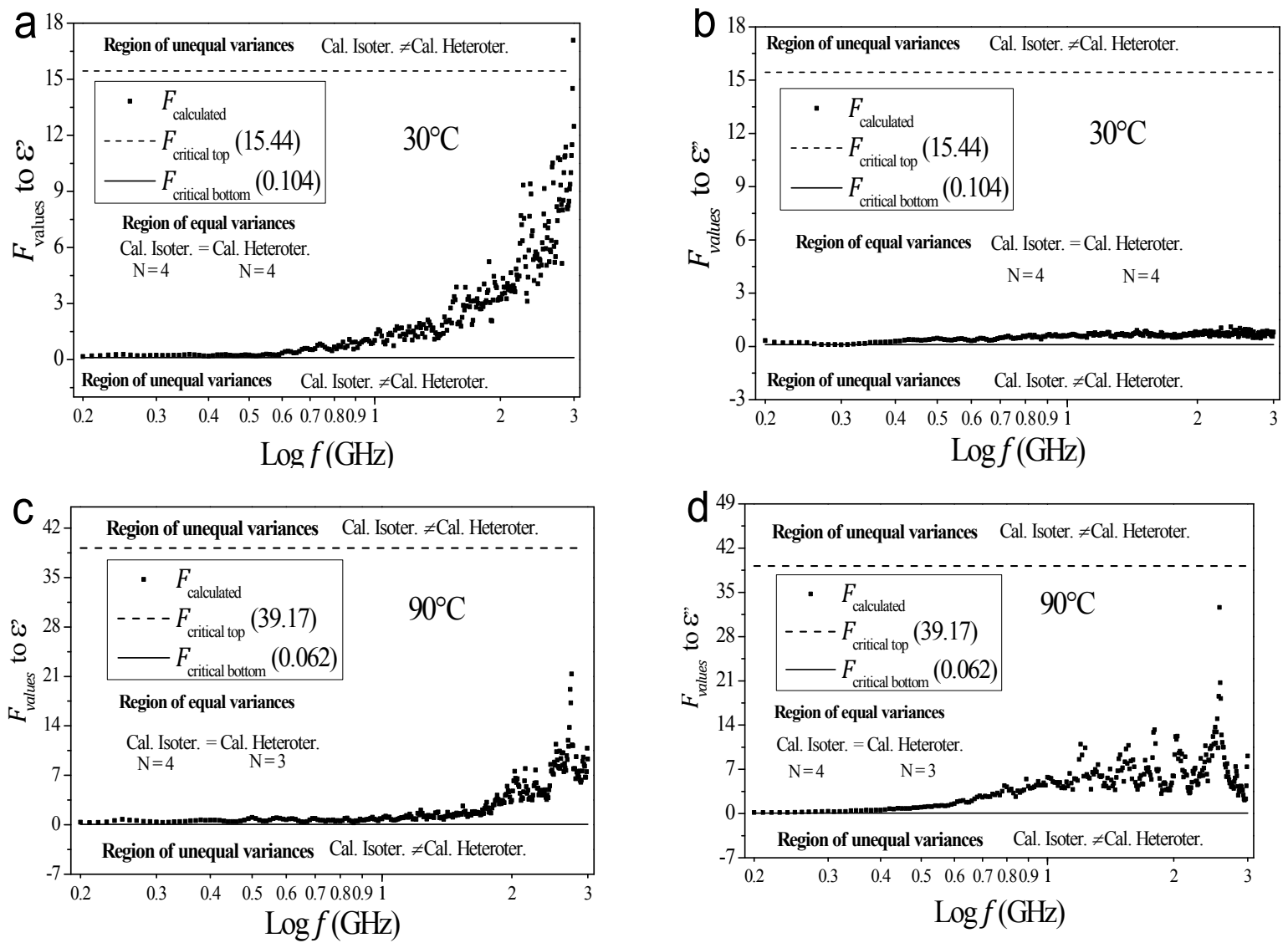

Fig. 5. F-test between isothermal and heterothermal variant 1 calibrations for (a) $\varepsilon^{\prime}$ and (b) $\varepsilon^{\prime \prime}$ at $30^{\circ} \mathrm{C}$, and (c) $\varepsilon^{\prime}$ and (d) $\varepsilon^{\prime \prime}$ at $90^{\circ} \mathrm{C}$.

Since variances are comparable, the statistical $t$-test for equal variances can be applied. Fig. 6 shows the results of the two-sided $t$-tests for 30 and $90^{\circ} \mathrm{C}$, indicating that the isothermal and heterothermal variant 1 calibration procedures are statistically equivalent.

\section{Comparison of Heterothermal Variant 1 and Heterothermal Variant 2 Calibrations}

Based on the results from sections III-B and III-C, the heterothermal variant 1D and heterothermal variant 2 calibrations were compared statistically. The thermal conditions of these variants are those in Table I. As previously done, the two-sided $F$-test was performed with the purpose of determining the equality of their variances. The number of replicates in both cases was $N=3$. Consider $\Delta \mathrm{T}_{\text {cal-meas }}$ as the difference between the temperature of measurement and the temperature of calibration, i.e. $\Delta \mathrm{T}_{\text {cal-meas }}=\mathrm{T}_{\text {cal }}-\mathrm{T}_{\text {meas }}$. Thus the values of $\Delta \mathrm{T}_{\text {cal-meas }}$ placed in the first column of Table III mean that the system was calibrated at $\mathrm{T}_{c a l}$ and measurements were made at temperatures $\mathrm{T}_{\text {meas }}$ from 10 to $50^{\circ} \mathrm{C}$ above $\mathrm{T}_{\text {cal }}$. For instance, since the calibration temperature for variant 2 was $20^{\circ} \mathrm{C}$, actual measurements were made from 30 to $70^{\circ} \mathrm{C}$. A graphical sample of the results is shown in Fig. 7 at $\mathrm{T}_{\text {meas }}$ of $30^{\circ} \mathrm{C}$, where it can be observed that most of the spectrum is in the region of equal variances. 

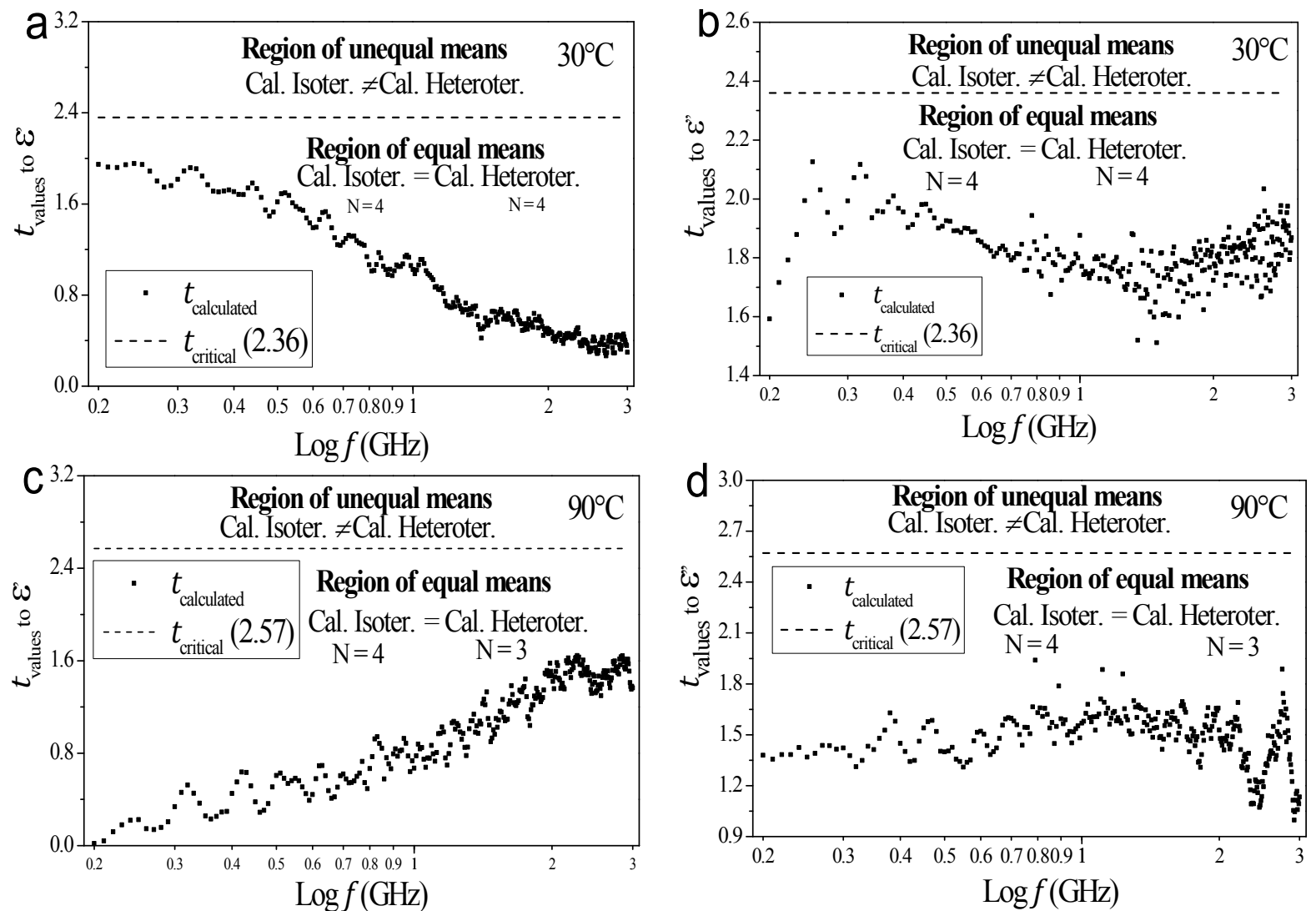

Fig. 6. $t$-tests between isothermal and heterothermal variant 1 calibrations for (a) $\varepsilon^{\prime}$ and (b) $\varepsilon^{\prime \prime}$ at $30^{\circ} \mathrm{C}$, and (c) $\varepsilon^{\prime}$ and (d) $\varepsilon^{\prime \prime}$ at $90^{\circ} \mathrm{C}$.
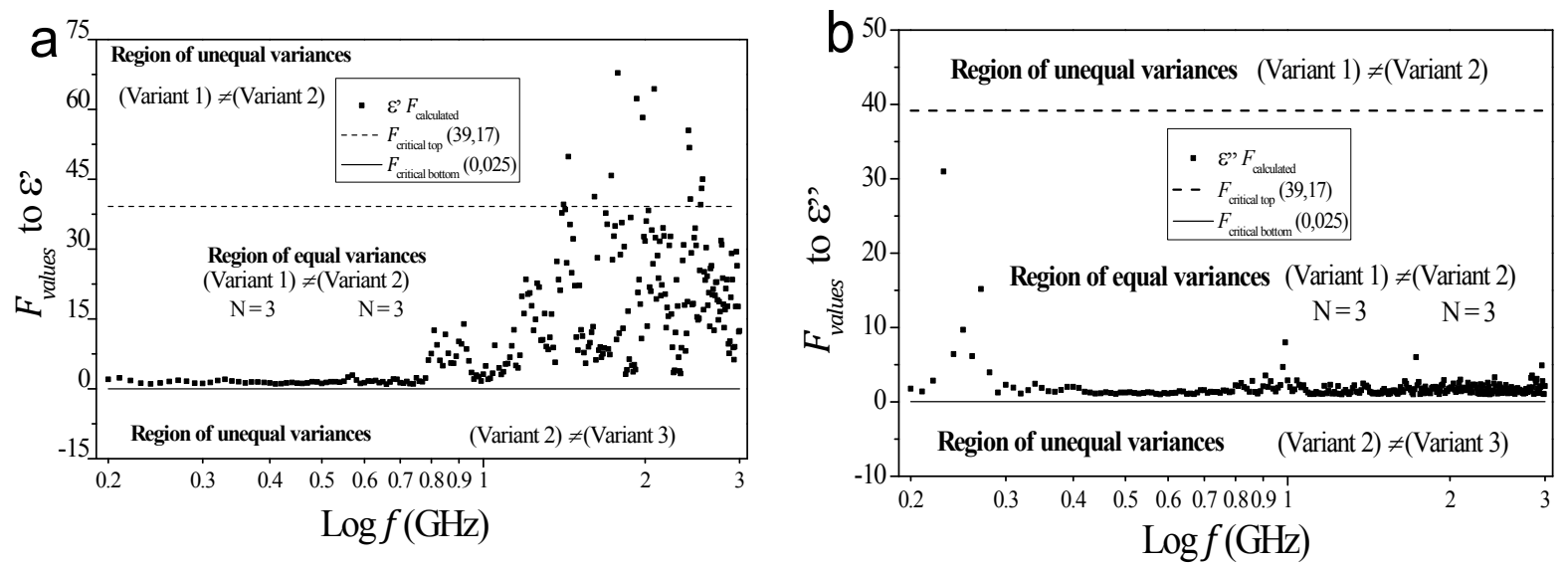

Fig. 7. $F$-test between the heterothermal variants $1 \mathrm{D}$ and 2 at the measurement temperature of $30^{\circ} \mathrm{C}$.

Table III shows the results for other temperatures. The total number of points along the frequency spectrum was 281 . In relation to this total, the results are given in percentage (\%) and absolute (Abs.) terms as the numbers of equal and unequal variances $\left(s^{2}\right)$. Thus $94 / 6$ means that $94 \%$ of the spectrum points have equal variances and only $6 \%$ exhibit different variances. If one observes all data for $\varepsilon^{\prime}$ and $\varepsilon^{\prime \prime}$, it may be conclude that all variances can be assumed to be equal, since at least $94 \%$ of the spectral data are identical. Therefore, both calibrations have equivalent precision. 
TABLE III. RESULTS OF $F$-TEST BETWEEN HETEROTHERMAL VARIANT 1 AND 2 CALIBRATIONS

\begin{tabular}{|c|c|c|c|c|}
\hline \multirow{3}{*}{$\Delta \mathrm{T}_{\text {cal-meas }}\left({ }^{\circ} \mathrm{C}\right)$} & \multicolumn{2}{|c|}{$\varepsilon^{\prime}$} & \multicolumn{2}{|c|}{$\varepsilon "$} \\
\hline & \multicolumn{2}{|c|}{$s^{2}$ equal $/ s^{2}$ unequal } & \multicolumn{2}{|c|}{$s^{2}$ equal $/ s^{2}$ unequal } \\
\hline & Absolute & Percentual (\%) & Absolute & Percentual (\%) \\
\hline 10 & $265 / 16$ & $94 / 6$ & $281 / 0$ & $100 / 0$ \\
\hline 20 & $278 / 3$ & $99 / 1$ & $280 / 1$ & $100 / 0$ \\
\hline 30 & $267 / 14$ & $95 / 5$ & $275 / 6$ & $98 / 2$ \\
\hline 40 & $276 / 5$ & $98 / 2$ & $270 / 11$ & $96 / 4$ \\
\hline 50 & $278 / 3$ & $99 / 1$ & $277 / 4$ & $99 / 1$ \\
\hline
\end{tabular}

After attesting the equality of variances, the statistics of the Student $t$-test for equal variances was used. The results are summarized in Table IV.

TABLE IV. RESULTS OF T-TEST BETWEEN HETEROTHERMAL VARIANT 1D AND 2 CALIBRATIONS

\begin{tabular}{|c|c|c|c|c|}
\hline & \multicolumn{2}{|c|}{$\varepsilon^{\prime}$} & \multicolumn{2}{|c|}{$\varepsilon^{\prime \prime}$} \\
\hline & \multicolumn{2}{|c|}{$\mathrm{n}^{\circ} t$ equal $/ \mathrm{n}^{\circ} t$ unequal } & \multicolumn{2}{|c|}{$\mathrm{n}^{\circ} t$ equal $/ \mathrm{n}^{\circ} t$ unequal } \\
\hline$\Delta \mathrm{T}_{\text {cal-meas }}\left({ }^{\circ} \mathrm{C}\right)$ & Absolute & Percentual (\%) & Absolute & Percentual (\%) \\
\hline 10 & $196 / 85$ & $70 / 30$ & $250 / 31$ & $89 / 11$ \\
\hline 20 & $273 / 8$ & $97 / 3$ & $261 / 20$ & $93 / 7$ \\
\hline 30 & $281 / 0$ & $100 / 0$ & $223 / 58$ & $79 / 21$ \\
\hline 40 & $269 / 13$ & $95 / 5$ & $263 / 18$ & $94 / 6$ \\
\hline 50 & $242 / 39$ & $86 / 14$ & $113 / 168$ & $40 / 60$ \\
\hline
\end{tabular}

The Student $t$-test presented better results for $\varepsilon^{\prime}$ than for $\varepsilon "$. This can be explained by the limitation of the spectral window to access the data, and by the fact that high frequency spectra to $\varepsilon^{\prime}$ and $\varepsilon^{\prime \prime}$ of many substances tend to converge in the tail region of the spectrum. This behavior is illustrated in Fig. 8.
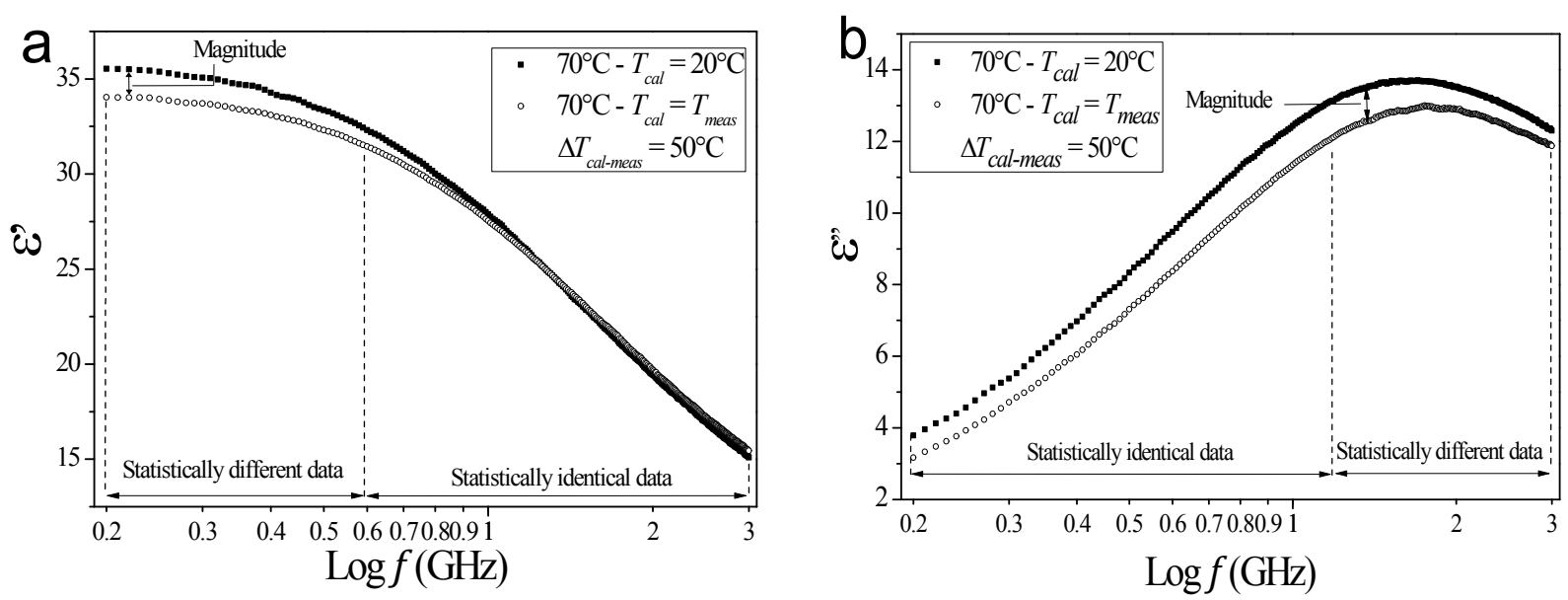

Fig. 8. Glycerol spectrum at $70^{\circ} \mathrm{C}$ with heterothermal variants $1 \mathrm{D}$ and 2 showing (a) $\varepsilon^{\prime}$ and (b) $\varepsilon^{\prime \prime}$. 
It can be seen in the figure that the curves behavior are identical, and the observed differences between the spectra are apparent in the magnitude values only, more specifically, in the regions for large values of $\varepsilon^{\prime}$ and $\varepsilon^{\prime \prime}$.

The fitting of these data to mathematical models to characterize the permittivity dependence with frequency [10] allows us to expand the spectral window for a more concise comparison. The Cole- Davidson model was used, since it is known in the literature as a good model to describe the dielectric behavior of glycerol [23], [24]. The four parameters required for the model, also referred to as the dielectric dispersion parameters, are: static permittivity $\left(\varepsilon_{S}\right)$, high-frequency permittivity $\left(\varepsilon_{\infty}\right)$, relaxation frequency $\left(f_{r}\right)$, and beta $(\beta)$. The Cole-Davidson model is written as

$$
\varepsilon=\varepsilon_{\infty}+\frac{\varepsilon_{S}-\varepsilon_{\infty}}{\left(1+j f / f_{r}\right)^{1-\beta}}
$$

Measurements were taken in triplicates and each measurement was fitted to the model. The resulting equations were used to carry out the descriptive statistics, $F$-test and Student's $t$-test. The process was repeated for each temperature and thermal variant. Preliminary curve fitting was used to provide the best values for $\varepsilon_{\infty}$ and $\beta$ at a given temperature, fixing them to get $\varepsilon_{S}$ and $f_{r}$.

Table $\mathrm{V}$ shows the values of the dispersion parameters for the heterothermal variants $1 \mathrm{D}$ and 2 (v. 1D and v. 2 in the table, respectively). The $t$-test results show that the relaxation frequency does not present significant statistical difference, confirming earlier observations that the main difference between calibrations is in the magnitude values of $\varepsilon^{\prime}$ and $\varepsilon^{\prime \prime}$, but not their position in the frequency spectrum. Since the magnitude values are mainly associated with $\varepsilon^{\prime}$, it is seen in the table that the results for $\mathrm{T}_{\text {meas }}$ up to $20^{\circ} \mathrm{C}$ above $\mathrm{T}_{\text {cal }}$ present no significant statistical difference.

TABLE V. DIELECTRIC DISPERSION PARAMETERS

\begin{tabular}{|c|c|c|c|c|c|c|c|c|}
\hline \multirow{2}{*}{$\Delta \mathrm{T}_{\text {cal-meas }}\left({ }^{\circ} \mathrm{C}\right)$} & \multicolumn{3}{|c|}{$\varepsilon_{S}$} & \multicolumn{3}{|c|}{$f_{r}(\mathrm{GHz})$} & \multirow[b]{2}{*}{$\varepsilon_{\infty}$} & \multirow[b]{2}{*}{$\beta$} \\
\hline & v. 1D & v. 2 & $t$-test & v. $1 \mathrm{D}$ & v. 2 & $t$-test & & \\
\hline 10 & 41.87 & 41.32 & Equal & 0.15 & 0.14 & Equal & 4.52 & 0.69 \\
\hline 20 & 39.72 & 40.65 & Equal & 0.28 & 0.26 & Equal & 4.51 & 0.70 \\
\hline 30 & 38.32 & 39.63 & Different & 0.50 & 0.46 & Equal & 4.84 & 0.71 \\
\hline 40 & 36.55 & 38.24 & Different & 0.77 & 0.75 & Equal & 4.45 & 0.70 \\
\hline 50 & 34.63 & 36.27 & Different & 1.32 & 1.22 & Equal & 3.84 & 0.69 \\
\hline
\end{tabular}

The temperature exerts an effect on the measured permittivity at two distinct points. The first is due to the measurement apparatus (basically the probe) and the second to the calibration standards. The origin of the first effect is the change in the coefficient of thermal expansion of the constituent materials of the probe. The second is the uncertainty regarding the dielectric properties of the calibration standards (essentially the RL).

Another general cause of measurement error is the difference between the permittivity of RL and MUT, such that the recommendation is to have their values as close as possible [9]. One such example is placed in [25] where the author measured Brazil nut oil (MUT) using water and cyclohexane as RL 
in two independent calibration and measurement cycles. It was observed that if the permittivity of the $\mathrm{RL}\left(\varepsilon_{\mathrm{RL}}\right)$ is greater than the MUT ( $\left.\varepsilon_{\mathrm{MUT}}\right)$, the measured value is larger than the true value, i.e., it presents a positive bias.

Considering the static permittivity, and based on our observations and available data from the literature, we have built Table VI. In general, the change of probe parameters with temperature produces a positive bias in the measured results, regardless $\mathrm{T}_{\text {cal }}>\mathrm{T}_{\text {meas }}$ or $\mathrm{T}_{\text {cal }}<\mathrm{T}_{\text {meas }}$. Differences between RL and MUT dielectric properties produce positive or negative bias depending on $\varepsilon_{S_{\mathrm{RL}}}>$ $\varepsilon_{S_{\mathrm{MUT}}}$ or $\varepsilon_{S_{\mathrm{RL}}}<\varepsilon_{S_{\mathrm{MUT}}}$, respectively.

TABLE VI. OBSERVED THERMAL EFFECT ON MEASURED PERMITTIVITY DATA

\begin{tabular}{|c|c|c|}
\hline Origin of effect & Condition & Result \\
\hline $\begin{array}{l}\text { Change in coefficient of thermal expansion of } \\
\text { the constituent materials of the probe }\end{array}$ & $\begin{array}{c}\mathrm{T}_{\text {cal }}> \\
\mathrm{T}_{\text {meas }} \\
\mathrm{T}_{\text {cal }}< \\
\mathrm{T}_{\text {meas }}\end{array}$ & $\begin{array}{l}\text { Measured value }>\text { True } \\
\text { value }\end{array}$ \\
\hline $\begin{array}{l}\text { Difference of dielectric property between RL } \\
\text { and MUT }\end{array}$ & $\begin{array}{c}\varepsilon_{S_{\mathrm{RL}}}> \\
\varepsilon_{S_{\mathrm{MUT}}} \\
\varepsilon_{S_{\mathrm{RL}}}< \\
\varepsilon_{S_{\mathrm{MUT}}}\end{array}$ & $\begin{array}{l}\text { Measured value }<\text { True } \\
\text { value } \\
\text { Measured value }<\text { True } \\
\text { value }\end{array}$ \\
\hline
\end{tabular}

Based on the static permittivity, and assuming the results from variant 1 as the true values, consider that the relative measurement error $\left(\Delta_{r} \%\right)$ is given according to equation (1).

$$
\Delta_{r} \%=\left|\frac{\varepsilon_{S_{\text {meas }}}-\varepsilon_{S_{T R U E}}}{\varepsilon_{S_{\text {meas }}}}\right| \times 100 \%
$$

Where, $\varepsilon_{S_{T R U E}}$ is the static permittivity obtained with variant $1 \mathrm{D}$ calibration and $\varepsilon_{S_{\text {meas }}}$ is the static permittivity obtained from variant 2 calibration. Fig. 9 shows the results acquired from equation (1) for $\Delta \mathrm{T}_{\text {cal-meas }}$ from 10 to $50^{\circ} \mathrm{C}$. The results show that in our experiment the relative error for the static permittivity values is less than $5 \%$ for $\Delta \mathrm{T}_{\text {cal-meas }}$ up to $50^{\circ} \mathrm{C}$.

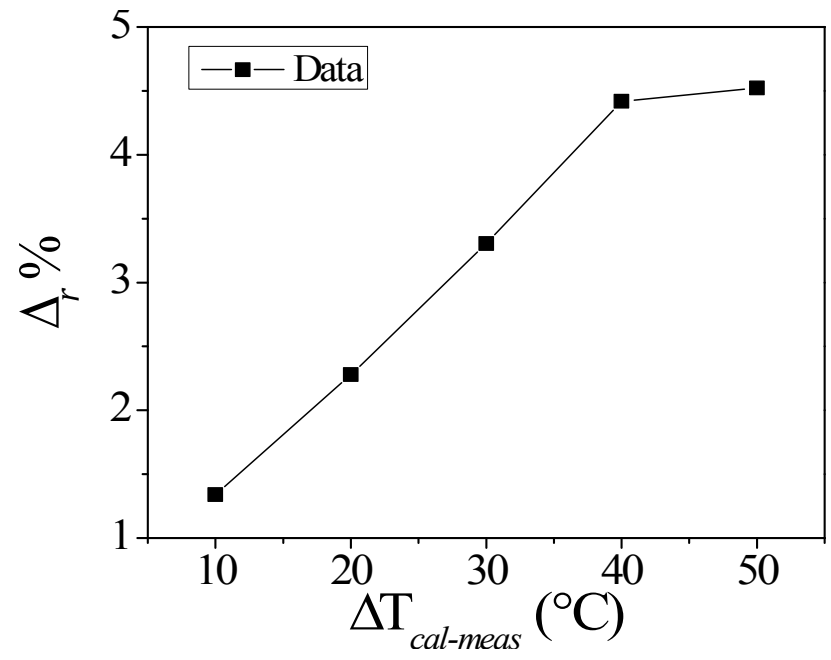

Fig. 9. Relative error between the static permittivity of variants 1 and 2 . 


\section{E. Heterothermal Variant 3 Calibration}

Heterothermal variant $3\left(\mathrm{~T}_{1}=\mathrm{T}_{2} \neq \mathrm{T}_{3} \neq \mathrm{T}_{\mathrm{MUT}}\right)$ is another very attractive calibration procedure. Studies in this respect are important to extend the thermal range of measurements. It is worth mentioning that there is no reported RL in the open literature that reaches the supra-threshold temperature of the used probe $\left(200^{\circ} \mathrm{C}\right)$. From the previous discussion, the more fundamental question here is to establish a temperature difference between $\mathrm{T}_{\text {cal }}$ and $\mathrm{T}_{\text {meas }} \quad\left(\mathrm{T}_{3}\right.$ and $\mathrm{T}_{\text {MUT }}$, respectively) where measurement errors are tolerable. For instance, Fig. 9 shows that for the static permittivity where $\mathrm{T}_{\text {cal }}$ differs by $50^{\circ} \mathrm{C}$ from $\mathrm{T}_{\text {meas }}$, the difference between the results obtained with variants 1 and 2 differs by less than $5 \%$ in absolute terms. Based on the accuracy of the coaxial probe method $( \pm 5 \%)$, and using air, short and water as standards, it can be assumed that variant 1 is equivalent to variant 2 with $\mathrm{T}_{\text {meas }}$ differing by up to $50^{\circ} \mathrm{C}$ from $\mathrm{T}_{\text {cal }}$. This means that, in thesis, we can extend these results for variant 3 , and calibrate the measurement system at $90-95^{\circ} \mathrm{C}$ (considering water as RL) and make measurements up to $140-145^{\circ} \mathrm{C}$. It is, in fact, what characterizes variant 3 , which is nothing more than a mixture of variants $1\left(\mathrm{~T}_{1}=\mathrm{T}_{2} \neq \mathrm{T}_{3}=\mathrm{T}_{\mathrm{MUT}}\right)$ and variant $2\left(\mathrm{~T}_{1}=\mathrm{T}_{2}=\mathrm{T}_{3} \neq \mathrm{T}_{\mathrm{MUT}}\right)$.

In order to dig deeper into this question, we can make a comparison with data from the literature. As previously, the Cole-Davidson model is used in order to facilitate the comparison of spectral data, since that a statistical comparison of point-to-point data is more difficult, considering that each researcher obtains their data in different intervals and frequency steps. Thus, the static permittivity is used for this purpose. The values estimated in this work with variants 1 and 2 are compared with data in the literature [23, [27], [28]. Table VII summarizes these data.

We see in Table VII that the values of $\varepsilon_{s}$ obtained in the present work are in close agreement with the values in the literature. This indicates that the measurements were made correctly and reinforces the observation that variant 1 and 2 calibrations provide small differences between measured values, at least for $\Delta \mathrm{T}_{\text {cal-meas }}$ up $50^{\circ} \mathrm{C}$.

TABLE VII. STATIC PERMITTIVITY DATA COMPARISON

\begin{tabular}{|c|c|c|c|c|c|}
\hline & \multicolumn{3}{|c|}{ References } & \multicolumn{2}{|c|}{ Present work } \\
\hline $\mathrm{T}\left({ }^{\circ} \mathrm{C}\right)$ & [27] & [23] & [28] & Var. 1 & Var. 2 \\
\hline 30 & 44.75 & 42.1 & 41.17 & 41.87 & 41.32 \\
\hline 40 & 42.95 & 39.7 & $39.61^{\mathrm{a}}$ & 39.72 & 40.65 \\
\hline 50 & 41.10 & 37.8 & $38.00^{\mathrm{a}}$ & 38.32 & 39.63 \\
\hline 60 & - & - & 36.48 & 36.55 & 38.24 \\
\hline
\end{tabular}

Based on the assessments made so far, new glycerol measurements were performed. The variant 1D with PCalMeas 1:1 were used for measurements from 30 to $90^{\circ} \mathrm{C}$. And variant 3 with PCalMeas 1:4 were employed for measurements from 100 to $140^{\circ} \mathrm{C}$. The measurements were done in triplicates and their results were submitted to the $Q$-test to determine the appropriate replicates. The measured data are presented in Fig. 10. 

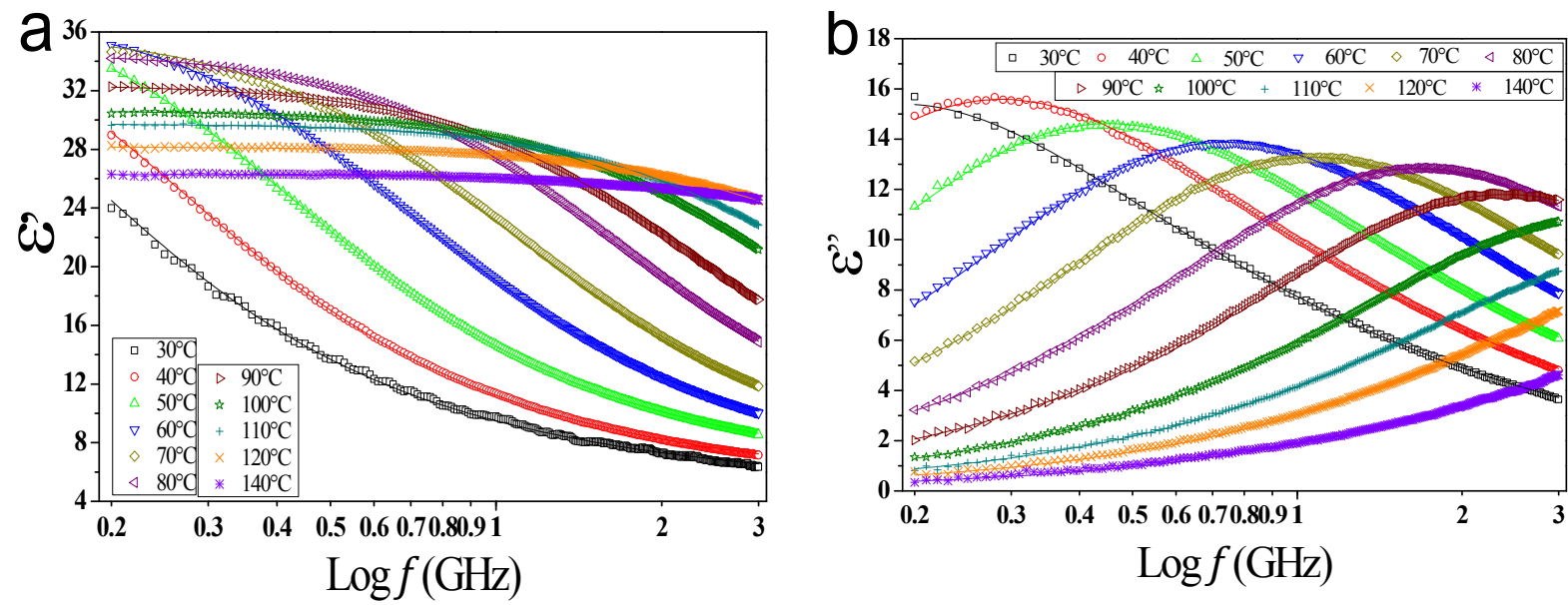

Fig. 10. Dielectric properties of glycerol from 30 to $140^{\circ} \mathrm{C}$ showing (a) real and (b) imaginary parts of $\boldsymbol{\varepsilon}$.

From the measured data, the Cole-Davidson model was used and the dielectric dispersion parameters of glycerol from 30 to $140^{\circ} \mathrm{C}$ were obtained.

These results are presented in Table VIII. Due to the narrow frequency band of our measurements, the values of $\beta$ for $\mathrm{T}_{\text {meas }} \geq 100^{\circ} \mathrm{C}$ were arbitrarily chosen. They are printed in gray in the Table VIII. Fitted curves for the measured data are represented by the full lines in Fig. 10.

\begin{tabular}{|c|c|c|c|c|}
\hline $\mathrm{T}\left({ }^{\circ} \mathrm{C}\right)$ & $\varepsilon_{S}$ & $f_{r}(\mathrm{GHz})$ & $\varepsilon_{\infty}$ & $\beta^{\mathrm{a}}$ \\
\hline $10^{\mathrm{b}}$ & 48.10 & 0.04 & $4 \pm 1$ & 0.65 \\
\hline $20^{\mathrm{b}}$ & 45.50 & 0.09 & $4 \pm 1$ & 0.69 \\
\hline 30 & 41.79 & 0.14 & 4.50 & 0.71 \\
\hline 40 & 39.77 & 0.22 & 4.74 & 0.72 \\
\hline 50 & 38.91 & 0.35 & 5.26 & 0.73 \\
\hline 60 & 37.38 & 0.59 & 5.39 & 0.75 \\
\hline 70 & 36.03 & 0.86 & 5.75 & 0.76 \\
\hline 80 & 34.64 & 1.38 & 5.64 & 0.78 \\
\hline 90 & 32.43 & 2.00 & 5.95 & 0.79 \\
\hline 100 & 30.65 & 2.65 & 2.65 & 0.63 \\
\hline 110 & 29.75 & 3.34 & 1.52 & 0.61 \\
\hline 120 & 28.17 & 4.19 & 7.38 & 0.60 \\
\hline 140 & 26.29 & 4.54 & 15.21 & 0.61 \\
\hline $\begin{array}{l}{ }^{\mathrm{a}} \text { Arbit } \\
{ }^{\mathrm{b}} \text { Refer }\end{array}$ & $\begin{array}{l}\text { hosen v } \\
3] \text {. }\end{array}$ & orintec & & \\
\hline
\end{tabular}

\section{FINAL CONSIDERATIONS}

In this work we have used the coaxial probe method for measuring the dielectric permittivity of liquid materials at high temperatures. The primary application of the results of this work is for the measurement of reaction mixtures in a microwave assisted chemical reaction in liquid phase homogeneous.

Using air, short and water as standards, we have analyzed some set calibration procedures with different thermal conditions and compared them with the isothermal case with the aid of statistical analysis. The results indicate that the calibration using $\mathrm{T}_{1}=\mathrm{T}_{2} \neq \mathrm{T}_{3}=\mathrm{T}_{\mathrm{MUT}}$ (variant 1 ), where $\mathrm{T}_{1}, \mathrm{~T}_{2}$, and $\mathrm{T}_{3}$ are the calibration temperature of each standard and $\mathrm{T}_{\mathrm{MUT}}$ is the material under test (MUT) 
temperature, is equivalent to the isothermal case $\left(T_{1}=T_{2}=T_{3}=T_{M U T}\right)$. Both are statically equals to variant $2\left(\mathrm{~T}_{1}=\mathrm{T}_{2}=\mathrm{T}_{3} \neq \mathrm{T}_{\mathrm{MUT}}\right)$ for a difference between the temperature of measurement and the temperature of calibration $\left(\Delta \mathrm{T}_{\text {cal-meas }}\right)$ of up to $20^{\circ} \mathrm{C}$. To achieve higher temperature measurements (at temperatures above the available data for the standards for instance) we have used variant $3\left(\mathrm{~T}_{1}=\right.$ $\mathrm{T}_{2} \neq \mathrm{T}_{3} \neq \mathrm{T}_{\text {MUT }}$ ). We have shown in our experiment that variant 3 is statistically equivalent to the previous schemes and can be used with reasonable accuracy for $\Delta \mathrm{T}_{\text {cal-meas }}$ up to $50^{\circ} \mathrm{C}$.

Taking into account the number of calibrations and the number of measurements (whose proportion is defined as PCalMeas in this work), we have shown that PCalMeas $1: n$ is more precise than PCalMeas 1:1, but it is not necessarily more accurate. Thus, using statistical tests for outliers, we can use and preserve the advantages of the PCalMeas 1:n scheme. We have used the previous results to measure glycerol up to $140^{\circ} \mathrm{C}$. From the measured data we also derived its Cole-Davidson model parameters from 30 to $140^{\circ} \mathrm{C}$.

The authors are aware that this is an initial study on these topics, and other studies (in development), such as evaluations with different MUTs, will be necessary for a better understanding of the subject.

\section{ACKNOWLEDGMENT}

The authors wish to thank CAPES (Coordination for the Improvement of Higher Education Personnel in Brazil) for the financial support.

\section{REFERENCES}

[1] L. Zhang, P. Liu, X. Shi, F. You and X. Dong, "A comparative study of a calibration method for measuring the dielectric properties of biological tissues on electrically small open-ended coaxial probe", Int. Conference on Biomedical Engineering and Biotechnology, pp. 658-661, 2012.

[2] 2015 Keysight N1501A Dielectric Probe Kit $10 \mathrm{MHz}$ to $50 \mathrm{GHz}$, Technical Overview Available in: www.keysight.com Access date: $11 / 11 / 2017$

[3] G. A. Dimitrakis, M. George, M. Poliakoff, I. Harrison, J. Robinson, S. Kingman, E. Lester, A. P. Gregory and K. Lees, "A system for traceable mesurement of the microwave complex permittivity of liquids at high pressure and temperatures," Meas. Sci. Tec., vol. 20, pp. 1-6, 2009.

[4] S. B. Lee, R. L. Smith Jr., H. Inomata and K. Arai, "Coaxial probe and apparatus for measuring the dielectric spectra of high pressure liquids and supercritical fluid mixtures”, Rev. Sci. Inst., vol. 71, pp. 4226-4230, 2000.

[5] D. Berube, F. M. Ghannouchi and P. Savard, "A comparative study of four open-ended coaxial probe models for permittivity measurements of lossy dielectric/biological materials at microwave frequencies," IEEE Trans. Micro. Theory Tech., vol. 44, pp. 1928-1934, 1996.

[6] D. V. Blackham and R. D. Pollard, "An improved technique for permittivity measurements using a coaxial probe," IEEE Trans. on Ins. Meas., vol. 46, pp. 1093-199, 1997.

[7] Agilent 5965-7709E Applying Error Correction to Network Analyzer Measurements, 2002.

[8] Udo Kaatze, "Reference liquids for the calibration of dielectric sensors and measurement instruments," Meas. Sci. Technol., vol. 18, pp. 967-976, 2007.

[9] B. Clarke, A. Gregory, D. Cannell, M. Patrick, S. Wylie, I. Youngs and G. Hill A guide to the characterization of dielectric materials at $R F$ and microwave frequencies. National physical laboratory, The Institute of Measurement and Control, 2003.

[10] A. P. Gregory and R. N. Clarke Tables of the Complex Permittivity of Dielectric Reference Liquids at Frequencies up to 5 GHz. NPL Report MAT 23, National Physical Laboratory, 2012.

[11] W. J. Ellison, K. Lamkaouchi and J. M. Moreau "Water: A dielectric reference," J. Mol. Liq., vol. 68, 171-279, 1996.

[12] W. J. Ellison "Permittivity of pure water, at standard atmospheric pressure, over the frequency range $0-25 \mathrm{THz}$ and the temperature range 0-100 ${ }^{\circ}$ C," J. Phys. Chem. Ref. Data., vol. 36, pp. 1-18, 2007. 
[13] B. G. Colpitts "Temperature sensitivity of coaxial probe complex permittivity measurement: experimental approach," IEEE Trans. Mic. Theory Tech., vol. 41, pp. 229-233, 1993.

[14] A. P. Gregory and R. N. Clarke "Dielectric metrology with coaxial sensors," Meas. Sci. Technol., vol. 18, pp. 13721386, 2007.

[15] J. Chen, K. Pitchai, S. Birla, D. D. Jones, J. Subbiah and R. Gonzalez, "Development of a multi-temperature calibration method for measuring dielectric properties of food," IEEE Trans. Diel. Elec. Ins., vol. 22, pp. 626-634, 2015.

[16] J. C. A. Santos, M. H. C. Dias, A. P. Aguiar, I. Borges Jr. and L. E. P. Borges, "Using the coaxial probe method for permittivity measurements of liquids at high temperatures,” J. Mic. Opt. Elect. App., vol. 8, pp. 78-91, 2009.

[17] D. C. Campos, E. L. Dall'Oglio, P. T. Sousa Jr., L. G. Vasconcelos and C. A. Kuhnen "Investigation of dielectric properties of the reaction mixture during the acid-catalyzed transesterification of Brazil nut oil for biodiesel production," Fuel, vol. 117, pp. 957-965, 2014.

[18] M. Arai, J. G. P. Binner and T. E. Cross, "Correction of errors owing to thermal elongation of high temperature coaxial probe for microwave permittivity measurement," Elect. Lett., vol. 31, pp. 1138-1139, 1995.

[19] D. C. Campos, E. L. Dall'Oglio, P. T. Sousa Jr., L. G. Vasconcelos, C. A. Kuhnen, A. C. Silva, F. Ribeiro and V. Rodrigues "Measurement of dielectric properties and microwave-assisted homogeneous acid-catalyzed transesterification in a monomode reactor", The J. Phys. Chem. A, vol.119, pp. 8971-8980, 2015.

[20] D. A. Skoog, D. M. West, F. J. Holler and S. R. Crouch, Fundamentals of Analytical Chemistry, Ed. 9, Cengage Learning, 2013.

[21] J. L. Devore Probability and statistics for engineering and the sciences, Ed. 6, Cengage learning, 2006.

[22] D. Zwillinger and S. Kokoska CRC Standard probability and statistics tables and formulae, Chapman \& Hall/CRC, 2000.

[23] Udo Kaatze, "Hydrogen network fluctuations: Dielectric spectra of glycerol-ethanol mixtures," Chem. Phys., vol. 403, pp. 74-80, 2012.

[24] P. Lukenheimer, A. Pimenov, B. Schiener, R. Böhmer and A. Loidl, "High-frequency dielectric spectroscopy on glycerol," Euro. Lett., vol. 33, No. 8, pp. 611-616, 1996.

[25] D. C. Campos, Construction of a flow-through reactor for the production of ethyl biodiesel via homogeneous catalysis with microwave induction and study of dielectric parameters of the constituents and reaction, Msc Dissertation, Federal University of Mato-Grosso, Mato-Grosso, Brazil, 2012.

[26] J. Homelin, J. B. Mehl and M. R. Moldover "The static dielectric constant of liquid water between 274 and 418 K near the saturated vapor pressure," Inter. J. Thermo., vol. 19, no. 5, 1359-1380, 1998.

[27] D. R. Lide, CRC Handbook of Chemistry and Physics, Ed. 90th, CRC Press, 2010.

[28] R. J. Sengwa, V. Khatri, S. Choudhary and S. Sankhla, "Temperature dependent static dielectric constant and viscosity behaviour of glycerol-amide binary mixtures: Characterization of dominant complex structures in dielectric polarization and viscous flow processes," J. Mol. Liq., vol. 154, pp. 117-123, 2010.

\section{ERRATA}

\section{On page 13, third column and second row of Table VI, the result for the difference of dielectric property between RL and MUT and condition $\varepsilon \mathrm{S}$ RL $>\varepsilon$ S MUT was incorrectly given:}

Condition: $\varepsilon$ S RL $>\varepsilon$ S MUT $\quad$ Result: Measured value $<$ True value

\section{The correct result is:}

Condition: $\varepsilon$ S RL $>\varepsilon$ S MUT Result: Measured value $>$ True value 